\title{
Lexicographic Perturbation for Multiparametric Linear Programming with Applications to Control
}

\author{
C.N. Jones ${ }^{a}$ E.C. Kerrigan ${ }^{b}$ J.M. Maciejowski ${ }^{c}$ \\ ${ }^{a}$ Automatic Control Laboratory, ETH Zurich, Physikstrasse 3, Zurich, Switzerland \\ ${ }^{\mathrm{b}}$ Department of Aeronautics and Department of Electrical and Electronic Engineering, Imperial College London, Exhibition \\ Road, London, SW7 2AZ, UK \\ ${ }^{\mathrm{c}}$ University of Cambridge, Department of Engineering, Trumpington Street Cambridge, CB2 1PZ, UK
}

\begin{abstract}
Optimal control problems for constrained linear systems with a linear cost can be posed as multiparametric linear programs with a parameter in the cost, or equivalently the right-hand side of the constraints, and solved explicitly offline. Degeneracy occurs when the control input, or optimiser, is non-unique, which can cause chattering of the control input and overlap of the polyhedral regions of the explicit solution. This paper introduces a new and efficient approach to the computation of the solution to a multiparametric linear program with the parameter in the cost in the presence of degeneracy. Rather than solve the degenerate problem directly, we solve a lexicographically (symbolically) perturbed version of it that is guaranteed to be non-degenerate. We show that every optimal solution of the perturbed problem is an optimal solution to the original and that the perturbed solution is continuous, unique and defined over a set of non-overlapping polyhedral regions. Furthermore, we introduce a new method for computing the optimal solution in an adjacent region that is very efficient in all cases, and reduces to a single simplex pivot for non-degenerate regions. The proposed algorithm is particularly suited for the calculation of the explicit solution to a class of constrained optimal control problems, since it ensures that the control input is everywhere continuous and unique, thereby removing the danger of chattering in problems with linear costs. The algorithm is compared through example to existing proposals and a significant complexity improvement is demonstrated.
\end{abstract}

Key words: Parametric Programming, Degeneracy, Parametric Optimization

\section{Introduction}

A multi-parametric linear program (mpLP) allows the data of a standard linear program to vary as a function of one or more parameters. mpLP solvers subdivide the set of feasible parameters into polyhedral regions in each of which the optimal solution is an affine function of the parameter. Once all such regions have been enumerated, the optimiser can then be computed for a given parameter by determining the region in which the parameter lies and evaluating the associated affine function. In this paper we consider the class of mpLPs that have parameters entering linearly into the cost or the right-hand side of the constraints, but not both simultaneously.

Although the original work on solving such problems by

\footnotetext{
* Corresponding author C.N.Jones.

Email: cjones@ee.ethz.ch

Tel: +41446328783
}

Gal and Nedoma [12] was done in the 1970s, there has recently been a resurgence of interest. This new work has been driven by the fact that the explicit solution to a class of constrained optimal controls problems can be computed using a multiparametric program $[3,4]$. There are currently three classes of algorithms for solving mpLPs. The original in [12] enumerates all optimal bases of the problem using a method derived from the simplex algorithm. A similar algorithm was proposed in [19] in which all of the bases of the dual-constraints are enumerated. A geometric approach has been proposed $[3,8]$ that directly explores the set of feasible parameters in a recursive manner, subdividing the feasible parameter space into so-called critical regions, in which the set of active constraints at the optimiser does not change. This method can introduce a large number of artificial cuts in the parameter space and an extension of this algorithm in $[1,14,22]$ addresses this problem by enumerating the regions in a non-recursive manner by stepping a sufficiently small distance over the facets of 
each region to find a point in a neighbouring region.

A primary problem for all mpLP algorithms is degeneracy. When an mpLP is degenerate, there is no longer a unique mapping from the parameter to the optimiser and the polyhedral subdivision is non-unique. These properties can result in overlapping regions and discontinuous optimisers. Both of these effects are detrimental to optimal control problems, since the optimiser is the control input and non-unique or discontinuous state-to-input maps can cause chattering of the actuators and lead to instability of the closed-loop trajectories. Further, algorithms based on direct exploration of the feasible space, such as $[1,14]$ cannot be guaranteed to find a cover for the set of feasible parameters, since degenerate regions do not necessarily meet facet-to-facet [20]. As a result, there may be regions of the parameter space for which no optimal solution has been computed.

The problem of degeneracy has been addressed in [21] by identifying regions of the parameter set that are degenerate and then solving a strictly convex multiparametric quadratic program (mpQP) over the set of optimal solutions in each such region. It is proven in [21] that the resulting polyhedral subdivision is non-overlapping and unique, and that the optimiser is both unique and continuous. However, this requires a secondary mpQP solver and, if the regions are not to overlap, several potentially expensive projection operations. It should be noted that it cannot be determined a priori whether the method [21] or that presented in this paper will result in a smaller number of regions and either situation may result depending on the specific problem.

In this paper, we introduce a new degeneracy handling approach for mpLPs based on lexicographic perturbation. Rather than solving the degenerate mpLP directly, we solve a lexicographic, or symbolic, perturbation of the given mpLP. We show that every optimal basis of the perturbed problem is also an optimal basis of the original and that the perturbed problem is guaranteed to be non-degenerate. Further, the optimal solution of the perturbed mpLP is continuous and the generated regions are both unique and their interiors are non-overlapping.

A new method of enumerating the solution based on $[1$, $14]$ is introduced that requires for each region the calculation of an LP of size equal to the degree of degeneracy of the region, and therefore requires only a single simple computation to find a non-degenerate adjacent region. While the proposed approach is more efficient and numerically robust than existing methods, it should be noted that in the worst case the solution can require an exponential number of regions, and therefore no method can be sub-exponential in the worst case.

The paper begins with a brief background on the primal lexicographically perturbed simplex method. We then follow [11] to extend this approach to a simultaneous perturbation of the primal and dual problems to standard and to multiparametric linear programming and give the proofs to support the claims made of the perturbed problem above. We show how a modification of the enumeration technique of $[1,14]$ can be applied to a lexicographically perturbed mpLP and prove it correct and complete. Finally, examples are given that demonstrate the pertinent properties of the algorithm as well as its improved efficiency over existing approaches.

\section{Notation}

If $A \in \mathbb{R}^{m \times n}$ and $I \subseteq\{1, \ldots, n\}, R \subseteq\{1, \ldots, m\}$, then $A_{\bullet I}\left(A_{R \bullet}\right)$ is the matrix formed by the columns (rows) of $A$ indexed by $I(R)$. If $c \in \mathbb{R}^{n}$ is a vector then $c_{I}$ is the vector formed by the elements of $c$ indexed by $I$. The set of affine combinations of points in a set $S \in \mathbb{R}^{n}$ is called the affine hull of $S$ and is denoted aff $(S)$. The dimension $\operatorname{dim}(S)$ of a set $S \subseteq \mathbb{R}^{n}$ is the dimension of aff $(S)$. If $\operatorname{dim}(S)$ is equal to $n$, then the set is called full-dimensional. The closure of a set $S$ is denoted $S^{+}$. A polyhedron is the intersection of a finite number of closed halfspaces. If $P=\{x \mid A x \leq b\}$ is a polyhedron and $H=\left\{x \mid a^{T} x \leq b\right\}$ is a halfspace such that $P \subseteq H$, then $P \cap\left\{x \mid a^{T} x=b\right\}$ is a face of $P .0$ and $\operatorname{dim} P-1$ faces are called the vertices and facets respectively.

\section{Preliminaries}

Consider the following linear program:

$$
\min _{x}\left\{c^{T} x \mid A x=b, x \geq 0\right\},
$$

where the cost is defined by the vector $c \in \mathbb{R}^{n}$, and the optimiser $x \in \mathbb{R}^{n}$ is restricted to lie in the polyhedron defined by the matrix $A \in \mathbb{R}^{m \times n}$ and the vector $b \in \mathbb{R}^{m}$.

Any set $B \subset\{1, \ldots, n\}$ such that $|B|=m$ and $\operatorname{rank} A_{\bullet B}=m$ is called a basis and we write $N=$ $\{1, \ldots, n\} \backslash B$ for its complement and call $x_{B}$ and $x_{N}$ the basic and non-basic variables respectively. Every basis $B$ defines a primal solution $x$ to the linear equations in (1), which is given by restricting the non-basic constraints to zero

$$
x_{B}=\beta b, \quad x_{N}=0,
$$

where we will use the standard notation $\beta \triangleq A_{\bullet B}^{-1}$ throughout. A basis is called primal feasible if the resulting primal solution also satisfies the inequality constraints in (1), $\beta b \geq 0$.

The reduced $\operatorname{cost}^{1}$ is defined as $\bar{c} \triangleq \kappa c$, where

$$
\kappa_{\bullet N} \triangleq I_{\bullet N}, \quad \kappa_{\bullet B} \triangleq I_{\bullet B}-A^{T} \beta^{T}
$$

1 Note that the reduced cost is often defined as $c_{N}-$ $A_{\bullet N}^{T} \beta^{T} c_{B}$, since $\bar{c}_{B}$ is clearly zero. We include the zero elements in the definition here so that the indices are preserved, i.e. $\bar{c}_{i}$ is related to the $i^{\text {th }}$ component $x_{i}$, rather than to the $i^{\text {th }}$ component of $x_{N}$. 
and $I \in \mathbb{R}^{n \times n}$ is the identity matrix. The following theorem provides the standard optimality condition for LP (1).

Theorem 1 (Optimality condition) [5] If B is a feasible basis of $L P(1)$, and $x$ and $\bar{c}$ are the primal solution and associated reduced cost, respectively, then $x$ is an optimal solution if and only if $\bar{c} \geq 0$.

The dual of LP (1) is:

$$
\max _{\lambda}\left\{b^{T} \lambda \mid A^{T} \lambda \leq c\right\} .
$$

Every basis $B$ also defines a dual solution $\lambda=\beta^{T} c_{B}$, which is clearly feasible for (4) if and only if the reduced cost $\bar{c}$ is positive, since the reduced cost is the dual slack variables. Therefore, we have the standard result that a basis $B$ defines an optimal solution of LP (1) if and only if the primal and dual solutions are both feasible.

\section{$3 \quad$ Unique Optimal Basis}

In this section we will discuss a modification to the standard primal simplex algorithm that will ensure that there is a unique optimal basis. A similar approach is outlined in [11] for the more general case of linear complementarity programs. In this paper, we propose a specific procedure for the primal simplex algorithm, which is particularly suited to parametric programming, as will be seen in the following section.

We first review the general primal simplex algorithm. The algorithm begins from any primal feasible basis $B$ and iterates the three steps of Algorithm 3, called a pivot, until the dual becomes feasible. Note that we have left out tests for optimality and unboundedness for brevity.

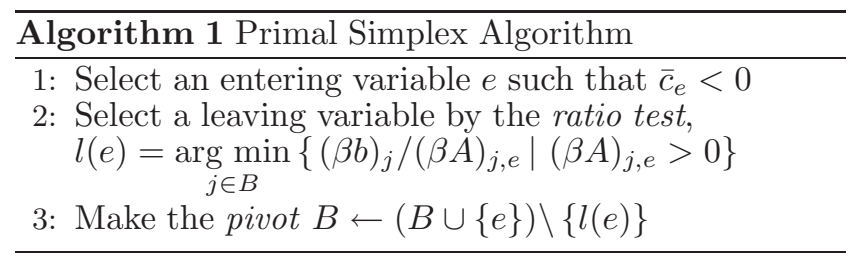

The goal of the algorithm is to bring the dual closer to feasibility with each iteration, until $\bar{c} \geq 0$, at which point the current basis is optimal by Theorem 1 . To this end, Step 1 selects an entering variable $e$ for which the dual is negative and allows the associated non-basic variable $x_{e}$ to increase from 0 . As the non-basic variable $x_{e}$ increases, the basic variables change as $x_{B}=\beta\left(b-A_{\bullet} x_{e}\right)$. The algorithm is required to maintain primal feasibility at all times and therefore the leaving variable $l(e)$ is chosen to ensure $\beta\left(b-A_{\bullet} x_{e}\right) \geq 0$, which gives rise to the ratio test.

A linear program is said to be primal degenerate if there is more than one basis that describes the optimal primal solution and dual degenerate if more than one primal solution is optimal. We now introduce a standard approach, called lexicographic perturbation, which removes the need to consider degeneracy. This technique is normally only applied to either the primal or to the dual for the purpose of preventing a problem called cycling. However, we here require a solution that is neither primal nor dual degenerate and so we follow [11] to extend the standard technique.

We will first review the standard primal lexicographic perturbation technique in the following section, and in Section 3.2 show how to extend the simplex algorithm to perturb both the primal and the dual simultaneously.

\subsection{Lexicographic Perturbation}

A vertex $x$ is called primal degenerate if more than $n-m$ constraints are active at $x^{2}$, where we recall that $A \in \mathbb{R}^{m \times n}$. When a vertex is primal degenerate, there is more than one feasible basis that can represent it. If $B$ is a primal-degenerate basis, then the basic variables are $x_{B}=\beta b$ of which some $x_{i}$ are equal to zero [18]. Early methods of resolving primal degeneracy consisted of adding a small random vector $\epsilon$ to the vector $b$, resulting in a primal solution $x_{B}=\beta(b+\epsilon)$, where all $x_{i}$ would be non-zero and therefore the basis could not be primal degenerate. Lexicographic perturbation is a more elaborate version of this simple idea and was originally proposed in [9].

Theorem 2 [18] There exists a positive number $\epsilon_{1}>0$, such that whenever $0<\epsilon_{0}<\epsilon_{1}$, the following perturbed problem is primal non-degenerate:

$$
\min _{x}\left\{c^{T} x \mid A x=b+\epsilon, x \geq 0\right\}
$$

where $\epsilon^{T} \triangleq\left[\begin{array}{llll}\epsilon_{0} & \epsilon_{0}^{2} & \ldots & \epsilon_{0}^{m}\end{array}\right]$

Remark 3 A perturbation is considered "sufficiently small" if it satisfies the conditions of Theorem 2. Note that the implementation of a lexicographic perturbation algorithm never requires the selection of a real-valued perturbation. Only the effect of a sufficiently small perturbation is considered and therefore there are no numerical issues or parameters to be chosen when perturbing the problem.

The relationship between bases of $\{x \mid A x=b, x \geq 0\}$ and of $\{x \mid A x=b+\epsilon, x \geq 0\}$ is given by the following theorem.

Theorem 4 [18] If $B$ is a feasible basis for the perturbed $L P(5)$ when $\epsilon$ is sufficiently small, then $B$ is a feasible basis for the unperturbed LP (1).

\footnotetext{
2 Note that $m$ is always assumed to be less than $n$, since there would otherwise be at most one solution to the equation $A x=b$ as the system of equations would be over-determined.
} 
Theorems 2 and 4 allow us to solve the non-primal degenerate problem (5) rather than the original degenerate one. The remainder of this section discusses the mechanics of solving a linear program over the perturbed polyhedron for a sufficiently small $\epsilon$.

Consider now a primal degenerate basis $B$ and recall that its inverse is denoted $\beta \triangleq A_{\bullet B}^{-1}$. The associated primal solution is then given by:

$$
\begin{aligned}
\left(x_{B}\right)_{i} & =(\beta b)_{i}+(\beta \epsilon)_{i} \\
& =\beta_{i \bullet} b+\beta_{i, 1} \epsilon_{0}+\beta_{i, 2} \epsilon_{0}^{2}+\cdots+\beta_{i, m} \epsilon_{0}^{m}
\end{aligned}
$$

Clearly, $\left(x_{B}\right)_{i}$ is primal feasible (i.e. positive) for all arbitrarily small $\epsilon_{0}$ if and only if the first non-zero element of the row vector $\left[\beta_{i} \bullet b \beta_{i}\right]$ is positive. This condition is referred to as lexico-positivity.

Definition 5 If $\gamma=\left(\gamma_{1}, \ldots, \gamma_{r}\right)$ is a vector, then it is said to be lexico-positive (or lex-positive) if $\gamma \neq 0$ and the first nonzero component of $\gamma$ is positive. Lexico positivity will be denoted by the symbol $\gamma \succ 0$. Given two vectors, $v$ and $u$, we say that $v \succ u$ if $v-u \succ 0$ and $v \preceq 0$ if $-v \succ 0$ or $v=0$. Given a set of vectors $\left\{v_{1}, \ldots, v_{r}\right\}$, the lexicographical minimum, denoted lexmin is the element $v_{i}$ such that $v_{j} \succ v_{i}$ for all $j \neq i$.

Clearly, not all feasible bases will satisfy the positivity condition of (6). The following definition introduces the bases of interest.

Definition 6 A feasible basis $B$ is called lexicographically feasible (lex-feasible) if every row of the matrix $[\beta b \quad \beta]$ is lex-positive.

Solving the lexicographically perturbed linear program (5) using a simplex approach is only slightly more complex than solving the unperturbed one. We now discuss a modification of the ratio test (Step 2 in Algorithm 3) that uses lexicographic perturbation. If $B$ is a lex-feasible basis and $e$ is the entering variable, then the lexicographic ratio test is given by:

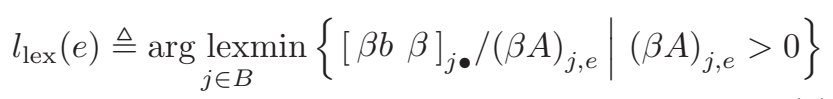

One can see that the lex-ratio test is derived in the same way as for the original. Given an entering variable $e$, the leaving variable $l_{\text {lex }}(e)$ is chosen to ensure that the new basis will still define a primal lex-feasible solution after $x_{e}$ is allowed to increase: $x_{B}=\beta b-\beta A_{\bullet} x_{e}+\beta \epsilon \geq 0$. Note that the choice made by (7) must be unique since a non-unique solution would exist if and only if two rows of $[\beta b \beta]$ were identical, and this clearly cannot happen since $\beta$ is invertible by definition.

Remark 7 Note that the lex-ratio test (7) is computed without ever choosing a value for the variable $\epsilon$, but only the effect of a sufficiently small perturbation on the positivity of the solution $x_{B}$. Therefore, the computation does not involve small numbers, does not require the choice of any parameters and is numerically robust.

The following important theorem allows us to simply replace the ratio test of the primal simplex algorithm (Step 2) with the lexicographic ratio test (7) in the simplex algorithm and be sure that the optimal solution will be found and that it will not be primal degenerate. We will refer to a pivot made using the lexicographic ratio test as a lex-pivot.

Theorem 8 [18] If $B$ is a lex-feasible basis of $\{x \mid A x=b, x \geq 0\}$ and a lex-pivot is performed on $B$, the resulting basis will be lex-feasible. For sufficiently small $\epsilon$, there is a one-to-one correspondence between the vertices of $\{x \mid A x=b+\epsilon, x \geq 0\}$ and the lex-feasible bases of $\{x \mid A x=b, x \geq 0\}$. Two vertices $v_{1}$ and $v_{2}$ are adjacent if and only if there is a lex-feasible basis $B_{1}$ associated with $v_{1}$ and a lex-feasible basis $B_{2}$ associated with $v_{2}$ such that one can move from $B_{1}$ to $B_{2}$ (and vice versa) via a single lexicographic pivot.

Remark 9 An initial feasible lex-positive basis can be found by solving a modified LP using a lexicographic pivoting rule. Details can be found in [18].

\subsection{Dual Degeneracy}

In this section we further extend the standard lexicographic perturbation discussed previously to a method that simultaneously perturbs both the primal and the dual. Consider the following linear program:

$$
\min _{x}\left\{(c+\delta)^{T} x \mid A x=b+\epsilon, x \geq 0\right\},
$$

where $\delta$ and $\epsilon$ are lexicographic perturbations.

The primal, LP (1) is called dual-degenerate if and only if the dual (4) is primal degenerate [18]. If an LP is dualdegenerate, then there are multiple primal solutions that satisfy the optimality conditions. Therefore, if we lexicographically perturb the dual problem making the dual non-primal-degenerate, then the primal solution will be unique. Given a basis $B$, the primal and dual solutions of the perturbed problem LP (8) are given by:

$$
x_{B}=\beta b+\beta \epsilon \quad \lambda=\beta^{T} c_{B}+\beta^{T} \delta_{B}
$$

One can see that the primal solution $x$ depends only on $\epsilon$ and the dual $\lambda$ only on $\delta$. Therefore, we are free to perturb both the primal and the dual simultaneously and all theorems of the previous section will hold for both [11].

The goal of the simplex algorithm can now be re-stated as finding the unique optimal basis $B$ that is both primal and dual lex-feasible, where we recall that a basis is 
optimal if and only if it is both primal and dual feasible. We assume that an initial primal lex-feasible basis can be found (see [18] for an appropriate procedure), and that the simplex algorithm proceeds as before with the lex-ratio test ensuring that all pivots maintain primal lex-feasibility.

However, this lexicographic pivoting rule cannot be applied directly to the dual, as the intermediate bases in the primal simplex algorithm are not dual feasible. We instead change the selection of the entering variable (Step 1), by requiring the reduced cost to be lex-nonpositive, rather than negative. In such a way, the cost in the simplex algorithm decreases monotonically, and therefore with every pivot the system will get closer to dual lex-feasibility and optimality.

The reduced cost of LP (8) is $\bar{c}+\bar{\delta}=\kappa(c+\delta)$, where $\kappa$ is defined in (3). Therefore the reduced cost is lex-positive if and only if

$$
[\kappa c \kappa] \succ 0
$$

The primal simplex algorithm for simultaneous primal and dual lex-perturbation is given as follows:

\begin{tabular}{l}
$\overline{\text { Algorithm } 2 \text { Primal-Dual Lexicographically Perturbed }}$ \\
Primal Simplex Algorithm \\
\hline 1: Select an entering variable $e$ such that $[\kappa c \kappa]_{e \bullet} \preceq 0$ \\
2: Select leaving variable $l_{\text {lex }}(e)$ via lex ratio test $(7)$ \\
3: Make the pivot $B \leftarrow(B \cup\{e\}) \backslash\left\{l_{\text {lex }}(e)\right\}$ \\
\hline
\end{tabular}

\section{Lexicographically Perturbed Parametric Pro- gramming}

We now turn to the main point of this paper and apply lexicographic perturbation methods to parametric linear programming. It will be seen that uniqueness properties of the optimal basis for a lex-perturbed mpLP will allow the development of fast enumeration algorithms and will ensure continuity of the dual solution, which is of vital importance to control problems since it prevents chattering of the input that can lead to instability of the closed-loop trajectories.

We aim to solve the following perturbed mpLP in the parameter $\theta \in \mathbb{R}^{d}$ :

$$
J(\theta) \triangleq \min _{x}\left\{(E \theta+c+\delta)^{T} x \mid A x=b+\epsilon, x \geq 0\right\}
$$

'Solving' the multiparametric linear program consists of enumerating the optimal basis $B$ for every value of the parameter $\theta$ in some region $\Theta$, where we assume that there is a feasible solution for every $\theta \in \Theta$ and that the optimal solution is bounded.
Remark 10 As remarked in [8], a small amount of pre-processing will ensure that the region $\Theta$ is fulldimensional and that the projection of the dual constraints defines it: $\Theta=\left\{\theta \mid \exists y, A^{T} y \leq c+E \theta\right\}$.

In this section we will see that the set of parameters $\Theta$ can be sub-divided into full-dimensional polyhedral regions in which the optimal basis does not change. The mpLP can then be solved by enumerating all of these region-defining bases. The remainder of this section will discuss the properties of the solution to the perturbed mpLP and the next will outline an efficient enumeration technique.

Definition 11 Let $B$ be a primal lex-feasible basis of $m p L P(10)$. The set $R_{B}$ is defined as all $\theta$ such that $B$ is the lex-optimal basis of mpLP (10).

Remark 12 The above definition matches that from [12] and while it differs from that used in the more recent literature [3, 8, 15,21] both are equivalent for non-degenerate problems, as is the case here due to lex perturbation.

Lemma 13 Let $B$ be a primal lex-feasible basis of mpLP (10). The set $R_{B}$ is given by

$$
R_{B}=\{\theta \in \Theta \mid[\kappa(c+E \theta) \kappa] \succ 0\},
$$

where $\kappa$ is defined in (3).

PROOF. The basis $B$ is clearly primal lex-feasible for all values of the parameter. Equation 9 gives the condition for dual lex-feasibility and therefore optimality.

The following proposition shows that the sets $R_{B}$ are non-intersecting and that the set of all such regions partitions the set $\Theta$.

Proposition 14 If $B_{1}$ and $B_{2}, B_{1} \neq B_{2}$ are primal lexfeasible bases of mpLP (10), then $R_{B_{1}} \cap R_{B_{2}}=\emptyset$. If $\theta \in \Theta$, then there exists a region $R$ defined by (11) such that $\theta \in R$.

PROOF. For each value of $\theta$, there is exactly one primal lex-feasible basis that satisfies condition (9). The first result follows immediately. By assumption, the $\operatorname{mpLP}(10)$ is bounded and feasible for every $\theta \in \Theta$ and therefore an optimal basis $B$ exists for every $\theta$ and hence an appropriate region $R_{B}$ that contains $\theta$.

The sets $R_{B}$ are not necessarily full-dimensional, nor is the basis $B$ that is optimal in the relative interior of $R_{B}$ necessarily optimal on its boundary. For many applications, and in particular control applications where it is the control input, it is desirable to have a continuous dual solution. Therefore, we now turn to the dual solution and show that it is everywhere continuous. 
Lemma 15 Let $B$ be a primal lex-feasible basis of $m p L P(10)$. The closure of the set $R_{B}$ is given by

$$
R_{B}^{+}=\{\theta \in \Theta \mid \kappa(c+E \theta) \geq 0\}
$$

PROOF. From Lemma 13 , the set $R_{B}$ is given by $R_{B}=$ $\{\theta \mid \kappa(c+E \theta)+\kappa \delta>0\}$, where $\delta$ is a lexicographic perturbation. The result follows since $\delta$ can be taken arbitrarily small.

Theorem 16 Let $B_{1}$ and $B_{2}$ be primal lex-feasible bases for $m p L P(10)$. If the dual optimisers are $\lambda_{1}(\theta)$ and $\lambda_{2}(\theta)$ in $R_{B_{1}}$ and $R_{B_{2}}$ respectively, then $\lambda_{1}(\theta)=\lambda_{2}(\theta)$ for all $\theta \in R_{B_{1}}^{+} \cap R_{B_{2}}^{+}$, where $R^{+}$denotes the closure of $R$.

PROOF. Consider the following primal-perturbed parametric linear program:

$$
\min _{x}\left\{(E \theta+c)^{T} x \mid A x=b+\epsilon, x \geq 0\right\}
$$

where $\epsilon$ is a lexicographic perturbation. Let $B$ be a basis that is optimal for mpLP (13) for some value of $\theta$. The basis $B$ is clearly optimal in the closed region:

$$
\{\theta \mid \kappa(E \theta+c) \geq 0\}
$$

From Lemma 15, we can see that (14) is also the closure of $R_{B}$ for the fully perturbed mpLP (10). It follows that $B_{1}$ and $B_{2}$ are optimal for mpLP (13) in the region $R_{B_{1}}^{+} \cap R_{B_{2}}^{+}$. The mpLP (13) is primally lex-perturbed, and therefore not primal-degenerate by Theorem 2 . The dual solution is therefore everywhere unique.

The dual-solution of mpLP (13) and mpLP (10) are equal for a given basis, when $\delta$ is taken arbitrarily small. Since the dual solution of mpLP (13) is unique on the boundary of the closure, we have the result $\lambda_{1}(\theta)=$ $\lambda_{2}(\theta)$.

We will now restrict our attention to the enumeration of the bases $B$ that correspond only to full-dimensional regions $R_{B}$, since we can derive the optimal dual-solution on the boundaries from the above theorem. The next section will show how to compute neighbouring fulldimensional regions given an optimal basis that defines a full-dimensional region.

\subsection{Neighbourhood Problem}

The neighbours of a given full-dimensional region $R_{B}$ are defined as those bases $B^{\prime}$ such that $R_{B}^{+} \cap R_{B^{\prime}}^{+}$is a facet of both $R_{B}^{+}$and $R_{B^{\prime}}^{+}$. Given a basis $B$ and a facet $F$ of the region $R_{B}^{+}$, we will show in this section how to compute the optimal basis $B^{\prime}$ of the adjacent region that contains $F$.
The approach described in $[1,14]$ computes the adjacent region in three stages: first, a point $\theta_{\circ}$ is computed in the strict relative interior of the facet, second a small vector in a direction normal to the facet is added to $\theta_{\circ}$ and finally the mpLP is solved using an LP solver for this value of the parameter. The approach described here differs from this method in three ways. First, a point $\theta_{\circ}$ is never explicitly calculated in the relative interior of the facet, the computation of which can cause numerical errors and adds to complexity. Second, by adding a small vector normal to the facet to the point $\theta_{\circ}$, it is possible to 'step over' and miss small regions. The method described here poses an LP whose solution gives the optimal basis for an arbitrarily small step outside the region, ensuring no regions are missed. Finally, the optimal basis $B$ for the current region is primal lex-feasible for the LP whose solution gives the adjacent region. A result of this is that for non-degenerate regions, computing the adjacent optimal basis requires a single simplex pivot, rather than the solution of a complete high-dimensional LP.

Given a primal lex-feasible basis $B$ that defines a fulldimensional region $R_{B}$, the facets of the closure of the critical region $R_{B}$ are the $(d-1)$-dimensional faces of the polyhedron $R_{B}^{+}=\{\theta \mid \kappa(E \theta+c) \geq 0\}$ and each facet is either on the boundary of the region $\Theta$, or is the intersection of $R_{B}^{+}$with the closure of some other region. The neighbours of $R_{B}$ can then be determined in two steps: First, determine the facet-defining inequalities of $R_{B}^{+}$and second, for each facet, compute the basis that is optimal for a point just outside the facet.

All facets of the polyhedron $R_{B}^{+}$can be written as $R_{B}^{+} \cap$ $\left\{\theta \mid \kappa_{i}(c+E \theta)=0\right\}$ for some $i[23]$. The problem of determining which inequalities define facets of $R_{B}^{+}$comes down to redundancy elimination, or the removal of all redundant constraints from a polyhedron. A straightforward method of checking if the $i^{t h}$ constraint of the region $R_{B}^{+}$is redundant is to compute the optimal cost of the following LP [10]:

$$
J_{i}^{\star} \triangleq \min _{\theta}\left\{\begin{array}{l|l}
\kappa_{i} E \theta & \begin{array}{l}
\kappa_{j}(c+E \theta) \geq 0, j \neq i \\
\kappa_{i \bullet}(c+E \theta) \geq-1
\end{array}
\end{array}\right\}
$$

The $i^{t h}$ constraint is then redundant if and only if the optimal cost satisfies $J_{i}^{\star}+\kappa_{i \bullet} c \leq 0$.

The following theorem allows the computation of an adjacent region with a single linear program, for which a primal lex-feasible basis is already known.

Theorem 17 Let $B$ be a primal lex-feasible basis of mpLP (10), such that $R_{B}$ is full-dimensional and let $F \triangleq\left\{\theta \mid \kappa_{f} \bullet(E \theta+c)=0\right\} \cap R_{B}^{+}$be a facet of $R_{B}^{+}$. Let $B^{\prime}$ be the optimal basis of the following LP:

$$
\min _{x}\left\{\begin{array}{l|l}
\left(E E^{T} \kappa_{f \bullet}^{T}+\delta\right)^{T} x & \begin{array}{l}
A x=b+\epsilon, x \geq 0, \\
x_{\Gamma}=0
\end{array}
\end{array}\right\}
$$


where $\epsilon$ and $\delta$ are lexicographic perturbations and

$$
\begin{aligned}
& \Delta \triangleq\left\{i \mid \exists \alpha \geq 0, \alpha \kappa_{f} \bullet[E c]=\kappa_{i} \bullet\left[\begin{array}{ll}
E & c
\end{array}\right]\right\} \\
& \Gamma \triangleq\{1, \ldots, n\} \backslash \Delta
\end{aligned}
$$

If the minimum exists then the optimal basis $B^{\prime}$ defines the adjacent critical region $R_{B^{\prime}}$ such that $F=R_{B}^{+} \cap R_{B^{\prime}}^{+}$, otherwise $F$ is on the boundary of the region $\Theta$.

PROOF. Let $\theta_{\circ}$ be in the relative interior of the facet $F$. The goal is to find the optimal basis $B^{\prime}$ for mpLP (10) at a point $\theta=\theta_{\circ}+\gamma\left(\kappa_{f} \bullet E\right)^{T}$ for an arbitrarily small $\gamma>0$ (i.e. $\theta$ lies 'just outside' the region $R_{B}$ ). Two conditions must be satisfied: First, $B^{\prime}$ must be primal feasible and second, the reduced cost must be positive.

Consider the reduced cost of LP (10) at $\theta$ :

$$
\kappa^{\prime}\left(E \theta_{\circ}+c+E E^{T} \kappa_{f \bullet}^{T} \gamma+\delta\right) \geq 0
$$

where $\kappa^{\prime}$ is as defined in (3) for the basis $B^{\prime}$. From Theorem 16 we have that the dual-optimiser is unique on the intersection $R_{B}^{+} \cap R_{B^{\prime}}^{+}$. It follows that the unperturbed reduced cost for $\theta_{\circ} \in F$ for both $B$ and $B^{\prime}$ are equal, i.e. $\kappa\left(E \theta_{\circ}+c\right)=\kappa^{\prime}\left(E \theta_{\circ}+c\right)$.

By the definition of $\Gamma, \kappa_{\Gamma \bullet}^{\prime}\left(c+E \theta_{\circ}\right)>0$ and therefore $\kappa_{\Gamma \bullet}^{\prime}\left(E \theta_{\circ}+c+E E^{T} \kappa_{f \bullet}^{T} \bullet+\delta\right) \geq 0$ is satisfied for all $\delta, \gamma$ sufficiently small. Since $\kappa_{\Delta \bullet}^{\prime}\left(c+E \theta_{\circ}\right)=0$ for all $\theta_{\circ} \in F,(17)$ is equivalent to $\kappa_{\Delta \bullet}^{\prime}\left(E E^{T} \kappa_{f \bullet}^{T}+\delta / \gamma\right) \geq 0$. Therefore the basis $B^{\prime}$ satisfies the optimality conditions of mpLP (10) at the point $\theta$ if and only if $B^{\prime}$ is the optimiser of LP (16).

A standard simplex approach must first compute a feasible basis using a so-called Phase I LP and then proceed to compute the optimal basis from there. However, the known basis $B$ is already primal lex-feasible for the LP (16) and so Phase I can be avoided when using a primal simplex approach. The linear program is also generally of very small dimension, since $\Gamma$ is expected to contain most of the constraints. The following corollary shows that if the basis is in fact non-degenerate, then the adjacent basis $B^{\prime}$ is given by a single simplex pivot, since $\Gamma$ contains all constraints but one and those in the basis $B$.

Corollary 18 Let $B$ be a non-degenerate primal lexfeasible basis of mpLP (10) such that $R_{B}$ is fulldimensional and let $F \triangleq\left\{\theta \mid \kappa_{f}(E \theta+c)=0\right\} \cap R_{B}^{+}$be a facet of $R_{B}^{+}$. If the set $\Delta$ defined in Theorem 17 is the set $\{f\} \cup B$ then if $l_{\text {lex }}(f)$ is non-empty, the adjacent basis is given by $B^{\prime}=\left(B \backslash l_{\text {lex }}(f)\right) \cup\{f\}$, else $F$ is on the boundary of the region $\Theta$.
PROOF. The result follows directly from the definition of the simplex pivot (Section 3), by noting that there is only one non-basic variable that is not constrained to zero.

The algorithm given in the next section for the enumeration of all full-dimensional regions relies on the so-called facet-to-facet property [20].

Definition 19 (Facet-to-Facet) Let $P \triangleq\left\{P_{i} \mid i \in \mathcal{I}\right\}$ be a finite collection of full-dimensional polyhedra in $\mathbb{R}^{s}$, where $\operatorname{int}\left(P_{i}\right) \cap \operatorname{int}\left(P_{j}\right)=\emptyset$ for all $(i, j), i \neq j$. We say that the facet-to-facet property holds if $F_{(i, j)} \triangleq P_{i} \cap P_{j}$ is a facet of both $P_{i}$ and $P_{j}$ for all $(s-1)$-dimensional intersections $F_{(i, j)}, i \neq j$.

Corollary 20 If $R$ is the set of all full-dimensional regions $R_{B}^{+}$such that $B$ is a primal lex-feasible basis of mpLP (10), then $R$ satisfies the facet-to-facet property.

PROOF. LP (16) provides the adjacent basis given a region $R_{B}^{+}$and one of its facets $F$. Note that LP (16) is not a function of any point $\theta_{\circ}$ in the facet $F$. It follows that the adjacent optimal basis is the same for all $\theta_{\circ} \in F$. Since LP (16) is lexicographically perturbed, the solution is unique and this corollary follows directly.

\subsection{Region Enumeration}

In this section we introduce a graph whose enumeration provides the set of all optimal bases $B$ that define a fulldimensional region $R_{B}$.

Definition 21 The solution graph $\mathcal{G}$ of the mpLP (10) is defined as the pair $\mathcal{G} \triangleq(V, E)$. The nodes $\mathcal{V}$ are defined as the primal lex-feasible bases $B$ such that the region $R_{B}$ is full-dimensional. The pair $\left(B_{1}, B_{2}\right) \in \mathcal{V} \times V$ is in $\mathcal{E}$ if and only if $R_{B_{1}}^{+} \cap R_{B_{2}}^{+}$is a facet of both $R_{B_{1}}^{+}$and of $R_{B_{2}}^{+}$.

The graph clearly covers the set of all full-dimensional regions, and therefore its enumeration will provide the solution if it is connected.

Theorem 22 The graph $\mathcal{G}$ is connected.

PROOF. Consider any two points $\theta_{0}, \theta_{1} \in \Theta$ and the line $\theta=\theta_{0} \tau+(1-\tau) \theta_{1}, \tau \in[0,1]$ that connects them. Make the assumption that the line intersects only fulldimensional regions and their facets. If it does not, then an infinitely small perturbation of $\theta_{1}$ will ensure that it does as there are a finite number of lower-dimensional faces. Each facet that the line pierces clearly defines an $\operatorname{arc}$ in $\mathcal{G}$, and the entire sequence of arcs from $\theta_{0}$ to $\theta_{1}$ is also contained in $\mathcal{G}$ by construction. Since $\theta_{0}$ and $\theta_{1}$ are 
taken arbitrarily and the region $\Theta$ is convex, the graph must be connected.

All of the tools are now in place to enumerate the bases $B$ that define full-dimensional regions $R_{B}$. LP (15) determines which inequalities of a given region adjoin neighbours, and Theorem 17 computes those neighbours. Since the graph is connected, any standard enumeration technique, such as depth-first, will return all of the desired bases.

\section{$5 \quad$ Finite Horizon Optimal Control Examples}

The primary motivation for mpLPs in control is the calculation of so-called closed-form or explicit finite horizon optimal control laws. The goal is to control the following linear time invariant (LTI) system:

$$
\xi^{+}=\mathcal{A} \xi+\mathcal{B} u
$$

where $\xi \in \mathbb{R}^{n}$ is the state, $\xi^{+}$is the successor state and $u \in \mathbb{R}^{m}$ is the input. A standard finite horizon controller can be written as the solution to the following optimisation problem:

$$
\begin{aligned}
\underset{u_{0}, \ldots, u_{N-1}}{\operatorname{minimise}}\left\|Q_{F} \xi_{N}\right\|_{p} & +\sum_{i=0}^{N-1}\left\|R u_{i}\right\|_{p}+\left\|Q \xi_{i}\right\|_{p} \\
\text { subject to } \quad & \xi_{0}=\xi \\
\xi_{i+1} & =\mathcal{A} \xi_{i}+\mathcal{B} u_{i}, \quad i=0, . ., N-1 \\
\left(\xi_{i+1}, u_{i}\right) & \left.\in \mathcal{X}_{i+1} \times \mathcal{U}_{i}, \quad i=18\right)
\end{aligned}
$$

where $\xi_{i}$ and $u_{i}$ are future predicted states and inputs respectively, which are constrained to be in the sets $\mathcal{X}$ and $\mathcal{U}$, with the state at the end of the horizon $N$ required to lie in the terminal set $\mathcal{X}_{F}$. If the norm $p$ is either the $1-$, or $\infty-$ norm and the constraints $\mathcal{X}$ and $\mathcal{U}$ are polyhedra, then problem (18) can be written as an mpLP of the form considered in this paper, where the parameter is the current state $\xi$. See $[3,6]$ for details.

Degenerate Example If the mpLP is not degenerate for any value of the parameter, then the approach proposed in this paper will return the same result as all other current methods, as from Theorem 4 the perturbation has no effect in the non-degenerate case and we therefore here focus on a degenerate example. Consider the commonly used example of the discrete-time double integrator $[7,8,13,15]$ :

$$
\xi^{+}=\left[\begin{array}{ll}
1 & 1 \\
0 & 1
\end{array}\right] \xi+\left[\begin{array}{r}
1 \\
0.5
\end{array}\right] u,
$$

with the input and state constraints $|u| \leq 1,\|\xi\|_{\infty} \leq 5$ and a horizon $N$ of length 5 .
The problem (18) is made very degenerate by setting the weights $R, Q$ and $Q_{F}$ to zero. This situation arises naturally when variables are defined using zone objectives [16], such as in a surge tank where the level is unimportant so long as it doesn't under/over-flow.

The solution using the proposed lexicographic perturbation method is shown in Figure 1. Clearly, the regions $R_{B}$ join facet-to-facet, do not overlap and the input is continuous.

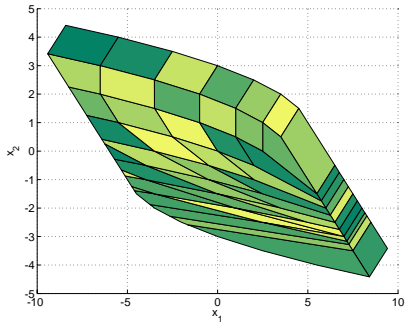

(a) Regions $R_{B}$

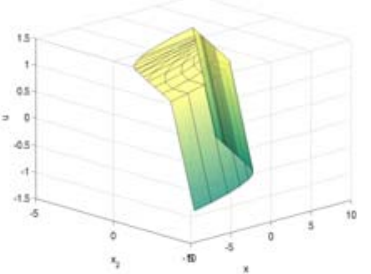

(b) Control Input
Fig. 1. Solution for degenerate Example

Random 3D System In this example we will investigate the performance difference between currently available mpLP codes and the efficiency benefit given by Theorem 17. Consider the following randomly generated system:

$\xi^{+}=\left[\begin{array}{rrr}-0.355 & 0.452 & -0.181 \\ 0.452 & -0.633 & -0.208 \\ -0.181 & -0.208 & -0.083\end{array}\right] \xi+\left[\begin{array}{rr}-1.007 & -0.999 \\ 1.598 & 0 \\ 1.055 & 1.426\end{array}\right] u$

with a prediction horizon $N=5$ and the constraints $\|u\|_{\infty} \leq 1,\|\xi\|_{\infty} \leq 5$ on the input and state respectively. The cost is the minimisation of the $\infty$-norm of the states and inputs at each point in time and the matrices $Q$ and $R$ are taken as the identity.

The efficiency of the three leading algorithms, implemented in MPT [15], the Hybrid Toolbox [2] and discussed in [8] were compared to the proposed lexicographic perturbation method. To ensure a fair comparison, the same LP code [17] was used in all four cases and the number of simplex pivots that each algorithm took were counted. The resulting data is presented in Table 1. The 'Pivots' columns show the number of lowdimensional simplex pivots for redundancy elimination and high-dimensional pivots for adjacency calculation that were taken. The improvement due to Theorem 17 can be seen in the small number of high-dimensional pivots required to compute adjacent regions.

\section{Acknowledgements}

The authors would like to thank Komei Fukuda for his valuable comments on this work. 


\begin{tabular}{l|r|r} 
Method & 3D Pivots & 20D Pivots \\
\hline Lex mpLP & 134,839 & 10,500 \\
MPT [15] & 161,702 & 70,167 \\
Hybrid Toolbox [2] & 319,208 & 147,954 \\
[8] & $1,392,740$ & 36,143
\end{tabular}

Table 1

Comparison of mpLP Methods

\section{References}

[1] M. Baotić. An efficient algorithm for multi-parametric quadratic programming. Technical report, ETH Zürich, Institut für Automatik, Physikstrasse 3, CH-8092, Switzerland, 2002.

[2] A. Bemporad. Hybrid toolbox, August 2005. Version 1.0.10, http://www.dii.unisi.it/hybrid/toolbox/.

[3] A. Bemporad, F. Borrelli, and M. Morari. Model predictive control based on linear programming - the explicit solution. IEEE Transactions on Automatic Control, 47(12):1974-1985, 2002.

[4] A. Bemporad, M. Morari, V. Dua, and E.N. Pistikopoulos. The explicit linear quadratic regulator for constrained systems. Automatica, 38(1):3-20, January 2002.

[5] D. Bertsimas and J.N. Tsitsiklis. Introduction to Linear Optimization. Athena Scientific, 1997.

[6] F. Borrelli. Discrete Time Constrained Optimal Control. $\mathrm{PhD}$ thesis, Swiss Federal Institute of Technology (ETH), October 2002.

[7] F. Borrelli. Constrained Optimal Control Of Linear And Hybrid Systems, volume 290 of Lecture Notes in Control and Information Sciences. Springer, 2003.

[8] F. Borrelli, A. Bemporad, and M. Morari. A Geometric Algorithm for Multi-Parametric Linear Programming. Journal of Optimization Theory and Applications, 118(3):515-540, September 2003.

[9] G.B. Dantzig, A. Orden, and P. Wolfe. The generalized simplex method for minimizing a linear form under linear inequality restraints. Pacific Journal of Mathematics, 5:183$195,1955$.

[10] K. Fukuda. Frequently asked questions in polyhedral computation. http://www.ifor.math.ethz.ch/fukuda/ polyfaq/polyfaq.html, October 2000.

[11] K. Fukuda, H.J. Lüthi, and M. Namkiki. The existence of a short sequence of admissible pivots to an optimal basis in LP and LCP. International Transactions of Operational Research, 4(4):273-384, 1997.

[12] Tomas Gal and Josef Nedoma. Multiparametric linear programming. Management Science, 18(7):406-422, 1972.

[13] P. Grieder. Efficient Computation of Feedback Controllers for Constrainted Systems. PhD thesis, Swiss Federal Institute of Technology (ETH), Zürich, 2004.

[14] P. Grieder, F. Borrelli, F. Torrisi, and M. Morari. Computation of the constrained infinite time linear quadratic regulator. Automatica, 40:701-708, 2004.

[15] M. Kvasnica, P. Grieder, and M. Baotić. Multi-Parametric Toolbox (MPT), 2004. http://control.ee.ethz.ch/ mpt/.

[16] J. M. Maciejowski. Predictive Control with Constraints. Prentice Hall, first edition, 2001.

[17] W. Murray and M. Saunders. Systems Optimization Laboratory (SOL). http://www.sbsi-sol-optimize.com.

[18] K.G. Murty. Linear Programming. John Wiley \& Sons, 1983.

[19] M. Schechter. Polyhedral functions and multiparametric linear programming. Journal of Optimization Theory and Applications, 53(2):269-280, May 1987.
[20] J. Spjøtvold, E.C. Kerrigan, C.N. Jones, P. Tøndel, and T.A. Johansen. On the facet-to-facet property of solutions to strictly convex multiparametric quadratic programs. Automatica, 2006. Accepted.

[21] J. Spjøtvold, P. Tøndel, and T.A. Johansen. A method for obtaining continuous solutions to multiparametric linear programs. In Proceedings of the $16^{\text {th }}$ IFAC World Congress, Prague, 2005.

[22] P. Tøndel, T.A. Johansen, and A. Bemporad. An algorithm for multi-parametric quadratic programming and explicit MPC solutions. Automatica, pages 489-497, 2003.

[23] G.M. Ziegler. Lectures on Polytopes. Springer-Verlag, New York, 1995. 